\title{
TRABALHO DE PARTO EXTENUANTE CULMINANDO COM RABDOMIÓLISE - RELATO DE CASO
}

\section{STRENUOUS LABOR CULMINATING IN RHABDOMYOLISIS - CASE REPORT}

\author{
Cristiano Salles Rodrigues ${ }^{1}$, Yohanna Peixoto Vilela ${ }^{2}$, Rafael de Castro Freitas ${ }^{3}$, Carlos Alexandre Ribeiro \\ Goulart $^{3}$, Juliana Batista Siqueira², Patrícia Guarino Barroso². \\ ${ }^{1}$ Coordenador da Residência de Ginecologia AFAMCI/FMC; Professor assistente da UFRJ Macaé/RJ; \\ Ginecologista e Obstetra \\ ${ }^{2}$ Residente em Ginecologia e Obstetrícia - AFAMCl, Campos dos Goytacazes/RJ. \\ ${ }^{3}$ Professor auxiliar da UFRJ Macaé/RJ; Ginecologista e Obstetra
}

\section{RESUMO}

Introdução: A rabdomiólise é uma síndrome resultante da desintegração do músculo estriado que cursa com mialgia, astenia e colúria, sua tríade clássica. Tem como causas mais comuns o estresse muscular excessivo. $\mathrm{Na}$ base de dados Scielo e Pubmed não foi encontrado literatura que compare o trabalho de parto ao exercício físico, mas considerando fatores inerentes ao trabalho de parto associados ao esforço físico, podemos inferir e caracterizá-lo como um exercício físico, visto que este também provoca estresse muscular. Objetivo: Relatar caso de rabdomiólise após trabalho de parto extenuante, com intuito de alertar sobre a prevenção, sintomas e seus fatores de risco, visando diagnóstico e tratamento precoces, melhorando o prognóstico das pacientes. Descrição do caso: Primigesta, 30 anos, 41 semanas e 5 dias, sem comorbidades, deu entrada no Hospital Unimed na cidade de Macaé/RJ com quadro de amniorrexe prematura. Após dezoito horas de trabalho de parto foi indicado cesariana por exaustão física, a pedido da paciente e de seu esposo. Durante sondagem vesical foi observada colúria, sendo realizada hidratação vigorosa com cristaloides e furosemida venosa, tendo o procedimento ocorrido sem intercorrências. Solicitados exames para elucidação diagnóstica, apresentou alterações que confirmou quadro de rabdomiólise. No pós-operatório apresentou quadro de intensa mialgia e astenia, tendo alta hospitalar no quinto dia pós-parto após melhora progressiva. Conclusão: A rabdomiólise deve ser considerada no diagnóstico diferencial de quadros de mialgia, astenia e colúria no pós-parto, permitindo seu tratamento imediato, reduzindo assim complicações renais e a comorbidade materna.

Palavras-chave: Rabdomiólise, Trabalho de parto extenuante, Colúria

\begin{abstract}
Introduction: Rhabdomyolysis is a syndrome resulting from the disintegration of the striated muscle that evolves with myalgia, asthenia and colúria, your classic triad. Have as common causes excessive muscular stress. On Scielo and Pubmed database not found literature that compare the labor to physical exercise, but considering factors inherent to the labor associated with the physical effort, we can infer and characterize it as a physical exercise, Since this also causes muscular stress. Objective: to report a case of rhabdomyolysis resulting in strenuous labor, in order to warn about the prevention, symptoms and their risk factors, seeking early diagnosis and treatment, thus improving the prognosis of the patients. Description of case: 30 years, first pregnancy, 41 weeks and 5 days, without Comorbidities, was admitted to the Unimed Hospital in the city of Macaé with amniorrexe frame premature. After 18 hours of labor was indicated cesarean section by physical exhaustion, at the request of the patient and of your spouse. During vesical probe colúria was observed, being carried out vigorous moisturizing with crystalloids and lasix, and venous procedure took place without complications. Ordered tests for diagnostic clarification, has tabled amendments that confirmed picture of rhabdomyolysis. Evolved postoperatively with intense frame myalgia and fatigue. Was discharged from the hospital on the 5 th day post partum after progressive improvement. Conclusion: Rhabdomyolysis should be considered as a differential diagnosis of myalgia, asthenia and colúria postpartum, allowing your immediate treatment reducing kidney complications and comorbidities Keywords: Rhabdomyolysis, Strenuous labor, Coluria
\end{abstract}




\section{INTRODUÇÃO}

A rabdomiólise é uma síndrome que varia de um quadro assintomático a uma condição de risco de vida associada a maiores aumentos na creatinofosfoquinase $(C K)^{1},{ }^{2},{ }^{3}$, desequilíbrio eletrolítico, insuficiência renal aguda (IRA) e coagulação intravascular disseminada ${ }^{5}$.

O exercício físico excessivo aparece como causa primária da rabdomiólise, mas devemos chamar atenção para as causas secundárias, que associadas ao exercício, possuem grande potencial de desenvolvimento e agravamento da síndrome, sendo as mais comuns o uso substâncias como drogas lícitas e ilícitas, anabolizantes, predisposição genética e fatores estressantes externos, como a temperatura e umidade do ambiente ${ }^{1},{ }^{2}, 4$.

Seu diagnóstico é baseado em padrões clínicos e laboratoriais, com colúria e mioglobinúria, atividade sérica de CK cinco vezes acima do limite máximo normal, elevados níveis de lactato desidrogenase, alanina aminotransferase e diminuição da concentração basal de cálcio no soro, devido à alterações nos canais iônicos de membrana, os quais influenciam, inclusive, na bomba de sódio e potássio e na produção de adenosina trifosfato (ATP), levando à contração miofibrilar sustentada, esgotamento de eletrólitos e ATP e provocando ainda mais danos em cascata aos canais iônicos ${ }^{1}, 4,8$.

0 resultado dessas alterações é o quadro inflamatório, uma miólise em cascata, que se apresenta com mialgia, colúria e astenia intensa, sua tríade clássica ${ }^{1,5}$. Seu tratamento é baseado na hidratação venosa vigorosa, estimulo renal e reposição de eletrólitos, a depender do quadro clínico apresentado5.

Apresenta-se a seguir relato de caso de rabdomiólise associada ao trabalho de parto, confirmada pelo quadro clínico e exames laboratoriais.

\section{RELATO DE CASO}

Paciente de 30 anos, primigesta, 41 semanas e 5 dias, sem comorbidades, sendo acompanhada por seu esposo e doula, deu entrada no Hospital Unimed na cidade de Macaé com queixa de perda de líquido iniciada há duas horas.

Internada em trabalho de parto com 2 centrímetros de dilatação, colo $60 \%$ apagado, centralizado, bolsa rota com saída de líquido fluido tinto de mecônio, -3 Delee. No seu plano de parto não desejava que fosse tomada nenhuma medida não natural para acelerar o trabalho de parto.

Após dezoito horas de trabalho de parto, apresentava-se com $6 \mathrm{~cm}$ de dilatação, bolsa rota com saída de líquido tinto de mecônio, -1 Delee, sendo indicado cesariana por exaustão física, a pedido da paciente e de seu esposo.

No centro cirúrgico, após realização de anestesia, na sondagem vesical foi observada colúria. Durante cesariana, foi realizada hidratação vigorosa com cristaloides e furosemida $1 \mathrm{ml}$ venosa, para estimulo renal, tendo o procedimento ocorrido sem intercorrências.

Solicitados exames para elucidação diagnóstica, apresentou CK de 22.100 U/L, creatinofosfoquinase componente muscular (CKMB) de 159,55 UI/L, transaminase glutâmico oxalacética (TGO) de $1.054 \mathrm{UI} / \mathrm{L}$, transaminase glutâmico pirúvico (TGP) de $187 \mathrm{UI} / \mathrm{L}$, mioglobina $607 \mathrm{UI} / \mathrm{L}$, aldolase 173 UI/L, proteína C reativa (PCR) de $130 \mathrm{mg} / \mathrm{L}$, elementos e sedimentos anormais na urina (EAS) com hematúria maciça e duas urinoculturas negativas e leucograma normal.

Evoluiu no pós-operatório com quadro de intensa mialgia, associado a limitação para amamentar nas 36 horas de pós-parto. Apresentando melhora progressiva da mialgia de dos parâmetros laboratoriais, a paciente teve alta hospitalar no quinto dia pós-parto.

\section{DISCUSSÃO}

A rabdomiólise é uma síndrome resultante da desintegração do músculo estriado e da consequente liberação de constituintes das células musculares no liquido extracelular e na circulação, sendo a mioglobina um dos principais compostos. ${ }^{1},{ }^{3}$ Por ser fracamente ligada as globulinas do plasma, esta chega a urina em pequenas quantidades, exceto quando há capacidade de ligação excedida, fazendo com que esta mioglobina seja filtrada pelos glomérulos, chegue aos túbulos e cause injúria renal e levando ao aparecimento de colúria e mialgia, dois componentes da tríade da rabdomiólise. ${ }^{1}$

A sua incidência no exercício físico é muito variável, dependendo das características da população estudada. Alguns autores aceitam a existência de condicionante genética como fator de risco, mas esta não é uma hipótese bem elucidada. ${ }^{6}$

A necrose isquêmica, que pode ser consequente a alterações hemodinâmicas ou de hidratação, assume papel importante no início da 
lesão muscular, assim como a hipocalemia resultante da sudorese pode condicionar redução da irrigação sanguínea, tendo como resultado câimbras e necrose isquêmica, contribuindo também para rabdomiólise. ${ }^{1,5,8}$

Esta patologia tem como causas mais comuns o estresse muscular excessivo induzido por trauma, atividade física, infecções, toxinas, substâncias químicas ou doenças associadas. ${ }^{1},{ }^{2,3}$

Após pesquisa na base de dados Pubmed e Scielo não foi encontrado trabalho publicado, estudo ou literatura que compare o trabalho de parto ao exercício físico, mas considerando fatores de estresse emocional, alterações cardiovasculares e alterações de temperatura associados ao esforço físico do trabalho de parto, podemos inferir e caracteriza-lo como um exercício físico, visto que este também provoca estresse muscular.

De fato, nem todo exercício físico irá desencadear lesão muscular, no entanto a realização de treinos mal programados, de alta intensidade, sem preparo físico ou na presença de condições climáticas adversas, são fatores desencadeantes da rabdomiólise. A paciente em trabalho de parto acaba se submetendo a realização de esforço físico e mental, associado a fatores externos que podem desencadear lesão muscular.

Neste caso, um trabalho de parto de evolução lenta, seguido de cansaço físico extremo referido pela própria paciente, juntamente com a sudorese e alterações de temperatura corpórea inerentes ao trabalho de parto, pode desencadear a patologia apresentada.

Mesmo apresentando a tríade clássica de mialgia, astenia e colúria, muitos sintomas da rabdomiólise são inespecíficos, o que pode dificultar o diagnóstico precoce, visto que estão ausentes em $50 \%$ dos casos, principalmente na fase inicial da doença. ${ }^{1}{ }^{2},{ }^{5} \mathrm{E}$ embora laboratorialmente seja característica a tríade de elevação do $\mathrm{CK}$, urina escura e cilindros granulosos pigmentados, o diagnóstico deve permanecer assentado na suspeição clínica.

A enzima CK está presente em todo o músculo estriado. Quando as células musculares se desintegram, a CK é liberada na corrente sanguínea. ${ }^{2}$ Como a degradação geral e a remoção são lentas, a concentração de CK permanece elevada por muito mais tempo e de uma maneira mais consistente do que a da mioglobina. Consequentemente, a CK é mais confiável do que a mioglobina na avaliação da presença e intensidade dos danos aos músculos. ${ }^{1,2}$

Visto que o exercício físico resulta frequentemente em mioglobinemia, mioglobinúria e aumento de CK, podendo também levar a alterações de coloração da urina, devemos afastar causas que cursam com mesmo perfil de alteração, como hemólise, hepatite, malária, uso de drogas, traumatismo genito-urinário e nefropatias, as quais entram como diagnóstico diferencial da rabdomiólise. $1,7,8,9,10$

O caso relatado aborda justamente uma paciente que, após submeter-se a estresse físico extenuante, evoluiu com astenia e mialgia intensas, a ponto de dificultar locomoção e cuidados com o recém-nascido, levando a dificuldade de amamentação associada à fadiga. Além disso, na sondagem para realização da cesariana, apresentou colúria, mais um sinal clínico da doença, a qual teve diagnóstico fechado com a associação aos exames complementares que evidenciaram aumento de importante de CK, CKMB, enzimas hepáticas, mioglobina e aldolase.

Em pacientes com diagnóstico de rabdomiólise, institui-se tratamento baseado em hidratação venosa rigorosa, podendo inicialmente ultrapassar 10L/dia, visando evitar ou diminuir o dano renal, bem como estimulo renal, prevenindo a precipitação tubular e o clearence dos pigmentos. ${ }^{1,},{ }^{3}$, Logo, a hidratação precoce melhora a perfusão renal, minimiza a injúria por isquemia e aumenta o fluxo urinário, promovendo a depuração e os efeitos tóxicos da mioglobina, melhorando significativamente o prognóstico dos pacientes,1 assim como a paciente em questão, que recebeu tratamento adequado, apresentando melhora progressiva dos sintomas e recebendo alta médica no quinto dia pós-parto. ${ }^{9}$

Portanto, pode-se concluir que a prevenção se baseia em proporcionar adequada hidratação durante todo trabalho de parto, corroborando à boa prática obstétrica de se oferecer líquidos e atentar às condições ambientais relacionadas a calor e umidade. ${ }^{1,9}, 10$

Mais uma alternativa seria promover e estimular a prática de exercícios físicos leves antes e durante a gestação, com acompanhamento adequado e progressão gradual das atividades, melhorando o condicionamento físico e visando melhorar a redistribuição da irrigação sanguínea por aumento do débito cardíaco, melhorando o metabolismo da glicose e melhorando a perfusão 
renal, prevenindo assim a incidência de rabdomiólise em pacientes em trabalho de parto. ${ }^{1,}{ }^{3}$

\section{CONCLUSÃO}

Este relato de caso se torna relevante por trazer a rabdomiólise como diagnóstico diferencial para quadros de colúria, mialgia e astenia em pacientes durante o trabalho de parto.

Seu rápido diagnóstico é de suma importância para o início precoce do tratamento preconizado, evitando assim, a falência renal bem como aumento da comorbidade materna.

\section{REFERÊNCIAS:}

1- José Galvão, Luís Gusmão, Marília Possante. Insuficiência renal e rabdomiólise induzidas por exercício físico, Rev Port Nefrol Hipert 2003; 17 (4): 189-197.

2- Lucas Félix Rossi, Luiz Antônio Marcatto Ramos, Renata Ribeiro Ramos, Aurélio Rotolo da Costa Araújo. Rabdomiólise induzida por esforço físico intenso com altos níveis de creatinoquinase. Revista da AMRIGS, Porto Alegre, 53 (3): 269-272, jul.-set. 2009.

3- Warren, Jason D.; Blumbergs, Peter C.; Thompson, Philip D. (março 2002). Rhabdomyolysis: a review. Muscle \& Nerve. 25 (3): 332-347.

4- Vanholder, Raymond; Sever, Mehmet Sükrü; Erek, Ekrem; Lameire, Norbert (1 de agosto de 2000). Rhabdomyolysis. Journal of the American Society of Nephrology (em inglês). 11 (8): 1553-1561. The syndrome of rhabdomyolysis: Complications and treatment. European Journal of Internal Medicine. 19 (8): 568-574.

5- Chatzizisis, Yiannis S.; Misirli, Gesthimani; Hatzitolios, Apostolos I.; Giannoglou, George D. (dezembro 2008).

6- $\quad$ Gabow PA, Kaehny WD, Kelleher SP: O espectro da rabdomiólise. Medicine (Baltimore) $61: 141-152,1982$

7- Poels PJE, Gabreëls FJM: rabdomiólise: uma revisão da literatura. Clin Neurol Neurosurg 95 : 175 -192,1993.

8- Uchoa RB, Fernandes CR. Rabdomiólise induzida por exercício e risco de hipertermia maligna - relato de caso. Rev Bras Anestesiol.2003; 53:1:63.

9- Marta Sousa Moniz1, Maria Inês Mascarenhas1, Carlos Escobar1, Pedro Nunes1, Clara Abadesso1, Helena Loureiro1 , Helena Almeida. Rabdomiólise como manifestação de uma doença metabólica: relato de caso. Rev Bras Ter Intensiva. 2017;29(1):111-114.

10- Iara Baldim Rabelo1 , Caio Pasquali Dias dos Santos1, Eduardo Mares Caldeira1 , Fernando Amaral Junqueira Nóbrega1, Flávia Ferreira Martins1, Maísa Ribeiro de Souza1, Mariana Martins Ferreira1, Moara dos Santos Oliveira Rodrigues1, Rafaela Dias Machado1, Taís Justimiano1. Rabdomiólise com altos níveis de creatinofosfoquinase, sem evolução para insuficiência renal. Rev Soc Bras Clin Med. 2016 jan-mar;14(1):38-40. 


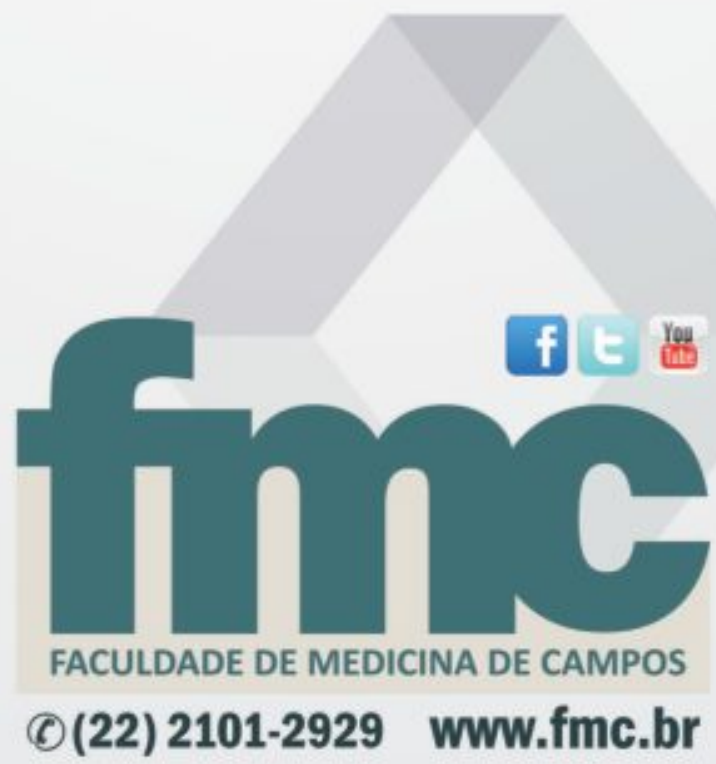

